\title{
Lapatinib: new opportunities for management of breast cancer
}

\author{
This article was published in the following Dove Press journal: \\ Breast Cancer:Targets and Therapy \\ II November 2010 \\ Number of times this article has been viewed
}

\author{
Julia Liao' \\ Michelle Gallas' \\ Mark Pegram ${ }^{1,2,3}$ \\ Joyce Slingerland 1,2,3,4 \\ 'Braman Family Breast Cancer \\ Institute, ${ }^{2}$ Division of Hematology/ \\ Oncology, University of Miami \\ Sylvester Comprehensive Cancer \\ Center, ${ }^{3}$ Departments of Medicine, \\ ${ }^{4}$ Biochemistry and Molecular Biology, \\ University of Miami Miller School of \\ Medicine, Miami, FL, USA
}

Correspondence: Joyce Slingerland Braman Family Breast Cancer Institute, UM Sylvester Comprehensive Cancer Center 1580 NW 10th Avenue, Batchelor 425, Miami, FL 33136, USA $\mathrm{Tel}+\mathrm{I} 3052436788$

Fax + I 3052434787

Email jslingerland@med.miami.edu
Abstract: Approximately $20 \%$ of new diagnosed breast cancers overexpress the human epidermal growth factor receptor 2 (EGFR2), also known as erythroblastic leukemia viral oncogene homolog 2 (ERBB2) protein, as a consequence of ERBB2 gene amplification, resulting in a poor prognosis. Clinical outcome can be substantially improved by ERBB2-targeted therapy. Lapatinib is a potent, orally bioavailable small molecule that reversibly and selectively inhibits epidermal growth factor receptor (EGFR1 or ERBB1) and ERBB2 tyrosine kinases. Lapatinib binds the adenosine triphosphate-binding site of the receptor's intracellular domain to inhibit tumor cell growth. This review summarizes the pharmacology, pharmacokinetics, efficacy, and tolerability of lapatinib, and reviews both Food and Drug Administration-approved and investigational uses of lapatinib in breast cancer therapy. The drug is generally well tolerated in patients, with diarrhea and rashes being the most common (usually mild or moderate) adverse effects. Unlike trastuzumab, lapatinib has infrequent adverse effects on cardiac function. Lapatinib has substantial activity for advanced ERBB2-positive breast cancer, particularly in combination with capecitabine, following progression after anthracyclines, taxanes, and trastuzumab. Lapatinib combined with capecitabine yielded significant improvements in time to progression and response rate compared with capecitabine alone. This drug can also be combined with letrozole for the treatment of postmenopausal women with ERBB2-positive breast cancer, for whom hormonal therapy is indicated. Lapatinib has shown early promise in treatment of central nervous system metastasis and is being further evaluated in various clinical settings.

Keywords: lapatinib, trastuzumab, ERBB family, ERBB2, breast cancer, capecitabine, letrozole

\section{Introduction}

Breast cancer is the second leading cause of cancer death amongst women in the US, and the most common form of cancer in women. In 2009, an estimated 194,280 new cases and 40,610 deaths resulted from breast cancer. ${ }^{1}$ As our knowledge and understanding of tumor cell biology has increased, adjuvant therapy has resulted in consistent incremental improvements in clinical outcome over the past decade. Despite these encouraging results in treating early breast cancer, the treatment of metastatic disease continues to pose formidable challenges. With metastatic breast cancer accounting for over 40,000 deaths each year in the US, novel therapeutic approaches to this pressing challenge are needed. ${ }^{1}$

Cancer is almost invariably linked to abnormal molecular circuitry, involving signaling pathways that control cell growth and differentiation. One crucial pathway that plays a central role in oncologic signaling in breast and certain other cancers begins 
with ERBB receptors, also known as epidermal growth factor receptors (EGFR). The ERBB family of receptor tyrosine kinases consists of four homologous members, ie, ERBB1 (EGFR, HER1), ERBB2 (HER2, c-ERBB2, neu), ERBB3 (HER3), and ERBB4 (HER4). ERBB receptors are crucial in organ development and regulating cellular differentiation and morphogenesis in a broad variety of tissues. ERBB1 and ERBB2 have been shown to promote the growth and survival of various types of epithelial malignancies. ERBB1 is overexpressed in up to $30 \%$ of human breast cancers, and ERBB2 is amplified and overexpressed in up to $20 \%$ of primary human breast cancers. ${ }^{2-5}$ ERBB1 overexpression has been documented in up to $60 \%$ of nonsmall cell lung cancer tumors. $^{6-9}$ ERBB3 has been implicated in the pathogenesis of multiple tumor types, including breast and ovarian cancers, while the ERBB4 gene is mutated in approximately $20 \%$ of melanomas. ${ }^{10,11}$

ERBB receptors are composed of an extracellular ligandbinding domain, a single transmembrane domain, and an intracellular tyrosine kinase domain. ERBB receptors exist in a predimerized state. ${ }^{12}$ When ligands such as epidermal growth factor, transforming growth factor- $\alpha$, amphiregulin, epiregulin, and heregulin bind to the predimerized state, a 2:2 ligand-to-receptor configuration is formed by the rotation of the transmembrane domain and subsequent rearrangement of each receptor subunit. ${ }^{13}$ Ligand binding to the extracellular domain ignites conformational rearrangements, triggering receptor dimerization to form ERBB homodimers and heterodimers that result in the recruitment and promotion of downstream signal transduction. The two best studied signaling pathways activated by this signaling cascade are the mitogen-activated protein kinase and the phosphatidylinositol 3-kinase (PI3K) pathways. The many tyrosine phosphorylation sites of ERBB3 serve as potent modules to activate intracellular signaling, especially the PI3K pathway. ${ }^{14}$ Amplification and/or mutation of the ERBB receptors results in the deregulation of downstream effector pathways contributing to the pathogenesis of many human malignancies. ${ }^{15}$

Members of the ERBB family, particularly ERBB1 and ERBB2, are commonly overexpressed, leading to deregulated proliferation and development, and contributing to oncogenesis in breast cancer. Immunohistochemical (IHC) studies have shown ERBB1 overexpression in up to $30 \%$ of primary breast cancers. ${ }^{3,4}$ This is germane to the understanding of ERBB2targeted therapies, because ERBB1 can heterodimerize with ERBB2. It is unclear what proportion of new breast cancers co-overexpress both ERBB1 and ERBB2 together. Up to
$20 \%$ of newly diagnosed breast cancers overexpress the ERBB2 gene due to gene amplification. ${ }^{5}$ When amplified, the ERBB2 gene leads to overexpression of $185^{\text {ERBB2 }}$, a $185 \mathrm{kDa}$ transmembrane glycoprotein and a well studied member of the ERBB family of receptor tyrosine kinases. ERBB2 amplification confers a particularly aggressive phenotype and poor prognosis, and therefore has been a prime target for the development of new breast cancer therapies. ERBB2 is the preferred binding partner for other ERBB receptor family members. Amplification of ERBB2 and resulting overexpression of the protein promotes spontaneous ligand-independent activation of the receptor's kinase domain.

ERBB2 overexpression status is routinely evaluated in pathologic breast cancer specimens. IHC is an immunostaining method whereby an antibody is targeted to ERBB2 expressed by tumor cells. The current guidelines for ERBB2 status are summarized as follows. A tumor is identified as having ERBB2 amplification (being ERBB2positive) either by showing uniform intense membrane staining in $>30 \%$ of invasive tumor cells on IHC (scored as $3+$ cell surface protein expression) or by a fluorescence in situ hybridization (FISH) result showing an amplified ERBB2 gene (average of $>$ six gene copies/nucleus for test systems without an internal control probe) or an ERBB2/ CEP17 ratio of more than 2.2, where CEP17 is a centromeric probe for chromosome 17 on which the ERBB2 gene resides. The false positive rate for ERBB2 scored as IHC 3+ (eg, that are actually not amplified) is small $(<4 \%)$ and it is currently standard of care to accept a $3+$ IHC as amplified without further testing. ${ }^{16-18}$ Patients are classified as having ERBB2-positive breast cancer based on positive results from either test. A negative ERBB2 test is defined as either an IHC result of 0 or $1+$ for cellular membrane protein expression (no staining or weak, incomplete membrane staining in any proportion of tumor cells), or a FISH result showing an ERBB2/CEP17 ratio of less than 1.8 or an average of fewer than four copies of the ERBB2 gene per nucleus for systems without an internal control probe. IHC for ERBB-2 scored as $2+$ requires further validation by FISH.

\section{Anti-ERBB2 therapies for breast cancer}

A number of new anticancer therapies have been developed to target breast cancers exhibiting ERBB2 amplification. The most important ERBB2-targeted therapies to date include monoclonal antibodies, antibody-drug conjugates, heat-shock protein-90 inhibitors, and tyrosine kinase inhibitors. ${ }^{19}$ Monoclonal antibodies function by binding to 
the extracellular domain of the receptor, recruiting cytotoxic lymphocytes and perturbing ERBB2-mediated signaling events to result in cell cycle arrest in $\mathrm{G}_{1}{ }^{20}$ The first monoclonal antibody to be clinically developed was trastuzumab (Herceptin ${ }^{\circledR}$; Genentech, Inc., San Francisco, CA).

These new classes of drugs targeting overexpressed ERBB2 have shown promising results in patients with ERBB2-positive breast cancers. Trastuzumab, a humanized monoclonal antibody that was approved by the US Food and Drug Administration (FDA) in 1998, is considered the backbone treatment for ERBB2-positive breast cancer. Trastuzumab's high specificity and affinity for the extracellular domain IV of the ERBB2 receptor has been shown to benefit patients with ERBB2-positive metastatic breast cancer when administered alone or in combination with chemotherapy, promoting tumor regression and increasing time to tumor progression and overall survival. ${ }^{21,22}$

Trastuzumab has also demonstrated significant activity in the adjuvant setting as a treatment for early ERBB2-positive breast cancer. ${ }^{23,24}$ Although the exact mechanism of action remains unknown, many have been proposed. Such mechanisms include inhibition of pathways involved in cell cycle progression, proteolytic cleavage of the ERBB2 receptor, inhibition of tumor angiogenesis and of DNA damage repair pathways, as well as of antibody-dependent cellular cytotoxicity.

The use of trastuzumab has become the standard of care for ERBB2-positive breast cancers in both the adjuvant setting and for metastatic breast cancers, but despite its benefits, a number of problems limit its clinical efficacy. Drug resistance, lack of penetration by trastuzumab of the blood-brain barrier with emergence of central nervous system metastasis, and cardiotoxicity are central among the limitations of trastuzumab in the clinical setting. Moreover, the need to administer trastuzumab intravenously adds to the cost and inconvenience of drug delivery. Both de novo and acquired resistance to trastuzumab have been observed. ${ }^{25}$ Most metastatic disease will progress within one year of beginning treatment, and recurrence has been observed with early breast cancer following adjuvant trastuzumab therapy. ${ }^{23,24,26,27}$ Although effective in inhibiting metastasis to the liver, lymph nodes, and bone, trastuzumab is associated with an increase in risk of central nervous system metastasis as a site of first tumor recurrence. Finally, incidences of cardiotoxicity had been observed in the pivotal Phase III trial evaluating trastuzumab in combination with chemotherapy. ${ }^{22}$ Cardiac dysfunction was identified in $27 \%$ of ERBB2-positive patients treated with trastuzumab, an anthracycline, and cyclophosphamide, and in $13 \%$ of those treated with paclitaxel and trastuzumab. These results have led to stringent monitoring of left ventricular ejection fraction in all patients on trastuzumab regimens. Therefore, there is a need for new agents that are more efficacious and better tolerated to target the ERBB2 family of receptors.

A new generation of trastuzumab-based drugs is currently in clinical trials. These antibody-drug conjugates, such as trastuzumab-DM1 (Genentech, Inc.) are a novel category of anti-ERBB2 therapeutics. T-DM1 consists of trastuzumab conjugated to DM1, a derivative of the antimicrotubule agent, maytansine. ${ }^{28}$ The trastuzumab domain directs the drug to ERBB2 where DM1 is then internalized to exert its cytotoxic effects. ${ }^{19}$

Pertuzumab is a monoclonal anti-ERBB2 antibody directed toward an epitope distinct from that targeted by trastuzumab, which interferes with receptor homo- and heterodimerization. It showed considerable efficacy in preclinical studies and good tolerability, bioavailability, and clinical activity upon three-weekly intravenous administration. ${ }^{29}$ While early clinical trials yielded disappointing results for pertuzumab in treatment of ERBB2-negative metastatic breast cancer, it may have a role together with trastuzumab in the treatment of trastuzumab-refractory, ERBB2-positive breast cancers. ${ }^{30,31}$

Heat-shock protein-90 plays a role in the proper folding of the ERBB2 protein following its biosynthesis in the ribosome. By inhibiting this chaperone function of heat-shock protein-90 with inhibitors such as tanespimycin (also known as 17-AAG), the stability of ERBB2 is undermined. ${ }^{32}$

Finally, small molecular tyrosine kinase inhibitors have been developed that block the nucleotide-binding site within the intracellular domain of ERBB proteins..$^{10}$ To date, only two ERBB2-targeted therapies, trastuzumab and lapatinib (Tykerb ${ }^{\circledR}$; GlaxoSmithKline, Research Triangle Park, NC), have been approved by the FDA for clinical use in ERBB2positive breast cancer patients. The reversible small molecule tyrosine kinase inhibitor, lapatinib, inhibits both ERBB1 and ERBB2, but its clinical utility for breast cancer is largely limited to ERBB2-positive disease. Since 2007, it has been approved by the FDA for use in combination with capecitabine (Xeloda ${ }^{\circledR} ;$ F. Hoffmann-La Roche, Basel, Switzerland) for the treatment of patients with advanced or metastatic breast cancers overexpressing the ERBB2 protein who have failed previous therapies. including anthracyclines, a taxane, and trastuzumab. ${ }^{33,34}$ This review will discuss certain advantages of lapatinib and its utility in the clinical setting, its pharmacology, pharmacokinetics, efficacy and tolerability, 
and briefly introduce some of the investigational new clinical studies of lapatinib.

\section{Pharmacology of lapatinib}

Lapatinib (originally known as GW572016) is a small molecule kinase inhibitor and derivative of 4-anilinoquinoline. It targets both ERBB1 and ERBB2 by reversibly attaching to the intracellular adenosine triphosphate binding site of the kinases. ${ }^{35,36}$ This inhibits the phosphorylation and subsequent activation of the PI3K-Akt and Ras-Raf-mitogen-activated protein kinase signaling cascades, increasing apoptotic activity and decreasing cellular proliferation (see Figure 1). ${ }^{35,36}$ Lapatinib has a large aniline quinazoline head group which allows access deep into the catalytic cleft of ERBB $1 .{ }^{37}$ This is a possible explanation for lapatinib's slow dissociation half-life of $>300$ minutes in comparison with other quinazolines, such as erlotinib (Tarceva ${ }^{\circledR}$; OSI/Genentech, Long Island, NY) and consequently longer inhibition of ERBB $1 .{ }^{37}$ The $50 \%$ inhibitory concentration is $<0.2 \mu \mathrm{M}$ for both ERBB1 and ERBB2, establishing lapatinib as a potent inhibitor.
Certain ERBB2-positive breast cancer lines express an amino terminally truncated carboxyl terminal fragment of ERBB2, p95 ${ }^{\text {ERBB2 }}$, which may arise either by proteolytic cleavage or by alternative translation of the ERBB2 mRNA transcript. ${ }^{38,39}$ These truncated ERBB2 isoforms retain potent tyrosine kinase activity, ${ }^{40}$ and lines are resistant to trastuzumab because p95 $5^{\text {ERBB2 }}$ lacks the extracellular binding domain to which trastuzumab binds. Although the biologic function and frequency of $\mathrm{p} 95^{\mathrm{ERBB} 2}$ expression in clinical ERBB2-positive breast cancers has not yet been exhaustively evaluated, this catalytically active truncated p95 $5^{\text {ERBB2 }}$ has been demonstrated in a number of studies of primary ERBB2 human breast cancers and may constitute a major mechanism of trastuzumab resistance. ${ }^{41,42}$ The extracellular domain of ERBB2 is commonly detected in the serum of patients with ERBB2-positive breast cancer, implying the presence of $\mathrm{p} 95^{\mathrm{ERBB} 2}$ in human disease. ${ }^{43-46}$

The expression of truncated ERBB2 isoforms is more common in metastatic than in primary ERBB2-positive breast cancers and is a predictor of worse outcome in metastatic breast cancer patients. ${ }^{41,42,47}$ Moreover, serum levels of

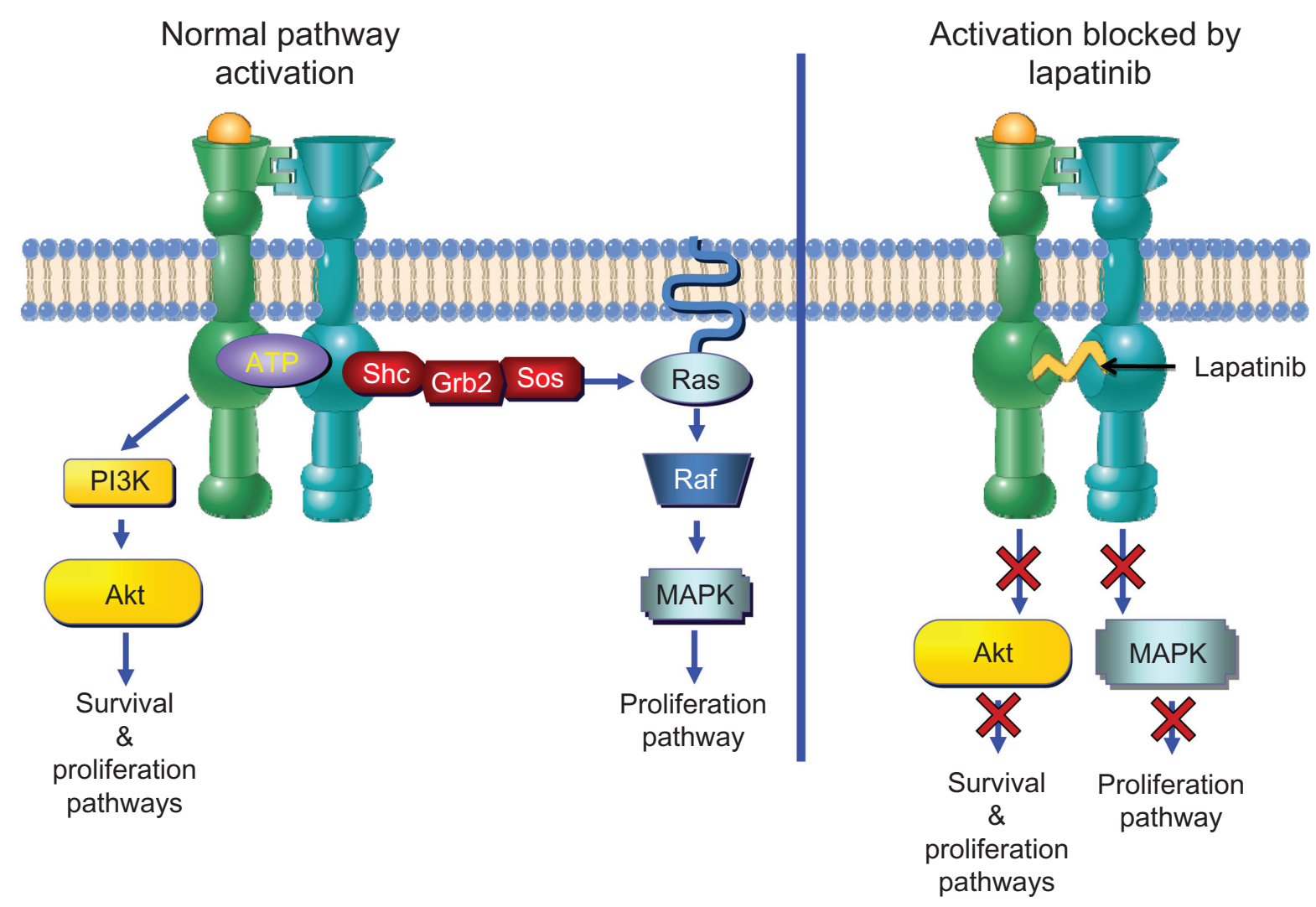

Figure I Mechanism of action of lapatinib. ERBB2 homodimerization or heterodimerization with other family members is promoted by binding of ligand (such as epithelial growth factor), and possibly by high receptor density secondary to ERBB2 amplification. Two key signaling pathways activated by receptor dimerization and activation are the PI3K-Akt pathway, which promotes both cell survival and cell cycle progression, and the mitogen-activated protein kinase (MAPK) pathway, which stimulates proliferation. Lapatinib blocks the catalytic cleft of the ERBBI and ERBB2 receptors, thereby preventing adenosine triphosphate binding and subsequent receptor phosphorylation leading to inhibition of downstream mitogenic signaling cascades. 
ERBB2 extracellular domain were associated with resistance to trastuzumab in a large multicenter study ${ }^{44}$ and the presence of truncated ERBB2 whether in serum or the tumor is also correlated with a decreased response to trastuzumab and poor outcome in patients with ERBB2-positive breast cancer. $^{40,43,48}$

In contrast with trastuzumab which cannot bind and inhibit $\mathrm{p} 95^{\mathrm{ERBB} 2}$, lapatinib binds to the catalytic cleft and can inhibit $\mathrm{p} 95^{\mathrm{ERBB} 2}$, and thus lapatinib can overcome $\mathrm{p} 95^{\mathrm{ERBB}}$ mediated trastuzumab resistance. Lapatinib has been shown to inhibit downstream mitogenic signaling cascades in cell lines expressing $\mathrm{p} 95^{\mathrm{ERBB} 2}$ and to block their growth in vitro and in vivo. ${ }^{40}$ Scaltriti et al demonstrated that treatment of p95 ${ }^{\mathrm{ERBB} 2}$-expressing breast cancer lines with lapatinib inhibited p95 ${ }^{\text {ERBB2 }}$ phosphorylation and reduced Akt and mitogen-activated protein kinase phosphorylation. These p95 ${ }^{\text {ERBB2}}$-expressing cell lines were trastuzumab-resistant but retained sensitivity to the antiproliferative effects of lapatinib. ${ }^{40}$ This study provides support for further characterization of ERBB2-positive breast tumors based on the presence or absence of $\mathrm{p} 95^{\mathrm{ERBB} 2}$ to help determine an optimal anti-ERBB2 therapy. Several other preclinical studies have also shown that lapatinib retains efficacy in trastuzumab-resistant cancer models. . $^{37,49,50}$

\section{Pharmacokinetics of lapatinib}

Bence et al evaluated the safety, tolerability, and pharmacokinetics of lapatinib as a single- and multiple-dose agent in a double-blind, randomized, placebo-controlled Phase I study. ${ }^{51}$ The results indicated that lapatinib was well tolerated in both regimens and not associated with any serious adverse events. In patients who received single doses, the most common adverse events were headache (23\%), rash (9\%), cough/ cold (6\%), diarrhea (4\%), and chalky taste (4\%). Of the 23 adverse events recorded in the 47 subject lapatinib dosing periods, only one subject had an adverse event greater than Grade 1. The multiple-dose study of lapatinib also reported that the drug was well tolerated with no serious adverse events. Gastrointestinal discomfort was the most common adverse event in $27 \%$ of the subjects who received lapatinib. Other adverse events included elevated liver function tests (17\%), rash (11\%), and headache $(6 \%)$. Of the 28 adverse events observed in subjects receiving either lapatinib or placebo, five were Grade 2 and resolved without treatment.

In this single-dose study, peak drug concentrations were reached approximately 3-4 hours after administration. The mean half-life was in the range 6-9 hours and increased as doses were raised. The multiple-dose study showed peak drug concentrations within 3-4 hours with no significant or consistent change over time. The average half-life was approximately seven hours on day 1 for all doses and 11 hours at $175 \mathrm{mg}$ on day 8 . Steady-state concentrations were achieved in six and seven days at the $100 \mathrm{mg}$ and $175 \mathrm{mg}$ doses, respectively.

The pharmacokinetics of lapatinib are summarized in the Table 1 . At the $1250 \mathrm{mg} /$ day dose approved by the FDA, the time to peak concentration $\left(\mathrm{T}_{\max }\right)$, peak concentration $\left(\mathrm{C}_{\max }\right)$, and area under the concentration-time curve (AUC) are 3-4 hours, $2.43 \mu \mathrm{g} / \mathrm{mL}$, and $36.2 \mu \mathrm{g} \cdot \mathrm{h} / \mathrm{mL}$, respectively. ${ }^{33,52-54}$ The effective half-life of daily dosing of lapatinib is 24 hours. The steady-state AUC is increased by approximately twofold if lapatinib is administered in divided daily doses compared with drug administration once daily at the same total dose..$^{33,53}$ Lapatinib is highly bound (>99\%) to albumin and $\alpha-1$ acid glycoprotein at $1 \mu \mathrm{mol} / \mathrm{L} .{ }^{33,53}$ In vitro studies revealed that lapatinib is a substrate for and an inhibitor of P-glycoprotein. ${ }^{33,53}$ Further, it has a broad distribution with a volume of distribution of $>2200 \mathrm{~L}$ that is significantly greater than the volume of body water. ${ }^{10,52,53}$ Elimination of lapatinib occurs through fecal excretion and hepatic metabolism via cytochrome P450 (CYP3A4, CYP3A5, CYP2C19, and CYP2C8), with 70\% through CYP3A4 ${ }^{33,53-55}$ Almost one-third (27\%) of an oral dose is recovered in feces and $<2 \%$ is recovered in urine with an elimination half-life of 14.2 hours in single-dose studies and approximately 24 hours with repeated dosing due to drug accumulation. ${ }^{33,53}$

Lapatinib is both a substrate and inhibitor of CYP3A4 and an inhibitor of CYP2C8 in vitro. A clinical study in healthy volunteers showed that when ketoconazole, a CYP3A4 inhib-

Table I Review of the pharmacokinetics of lapatinib at the dose of $1250 \mathrm{mg} /$ day, approved by the Food and Drug Administration

\begin{tabular}{ll}
\hline Absorption & Oral, not available in IV form \\
$T_{\max }$ & $3-4$ hours \\
Steady-state $C_{\max }$ & $2.43 \mu \mathrm{g} / \mathrm{mL}$ \\
AUC & $36.2 \mu \mathrm{g} \cdot \mathrm{h} / \mathrm{mL}$ \\
Distribution & Highly bound (>99\%) to albumin \\
Volume of distribution & $>2200 \mathrm{~L}$ \\
Metabolism & \\
Primary & CYP3A4 (70\% of metabolism) \\
Secondary & CYP3A5, CYP2CI9, CYP2C8 \\
Elimination & Primarily hepatic \\
Single dose $\mathrm{t}_{1 / 2}$ & $\mathrm{I} 4.2$ hours \\
Multiple doses $\mathrm{t}_{1 / 2}$ & 24 hours \\
\hline
\end{tabular}

Abbreviations: $t_{1 / 2}$, elimination half-life; AUC, area under the concentrationtime curve; IV, intravenous; $T_{\max }$, time to peak concentration; $C_{\text {max }}$ maximum concentration. 
itor, was administered, there was a 3.6-fold increase in the AUC of lapatinib and a 1.7-fold increase in elimination halflife relative to the control. ${ }^{33,53}$ By contrast, carbamazepine (Tegretol $^{\circledR}$; Novartis International AG, Basel, Switzerland), a CYP3A4 inducer, reduced the AUC of lapatinib by $72 \% .{ }^{33}$ Thus, strong CYP2C8 and CYP3A4 inhibitors (eg, grapefruit, clarithromycin, azole antifungals, and antiretrovirals) should be avoided to reduce risks of increased lapatinib plasma concentrations and, subsequently, lapatinib toxicity.

\section{Clinical trials \\ Monotherapy}

In 2008 Burstein et al reported the results of a multinational, open-label Phase II trial that evaluated the tolerability and efficacy of lapatinib monotherapy in patients with advanced or refractory metastatic breast cancer who had progressed on anthracycline, taxane, capecitabine, and trastuzumab therapies. ${ }^{56}$ The study comprised two cohorts: cohort A consisted of 140 patients with ERBB2-positive tumors; cohort B consisted of 89 patients with ERBB2negative tumors. Patients from each cohort received lapatinib $1500 \mathrm{mg}$ daily at a minimum of one hour before or after breakfast. Using the Response Evaluation Criteria in Solid Tumors, ERBB2-negative tumors from cohort B did not exhibit objective responses. In cohort $\mathrm{A}$, where patients' tumors were ERBB2-positive, there were three partial responses and three complete responses, leading to a response rate (complete response + partial response) of $4.3 \%$ by investigators' assessment or $1.4 \%$ (two partial responses) by independent review. ${ }^{56} \mathrm{~A}$ clinical benefit rate (complete response, partial response + stable disease $\geq 24$ weeks) was observed in $5.7 \%$ of the ERBB2-positive patients.

Independent review assessment of median time to tumor progression and median progression-free survival were similar for both ERBB2-positive and ERBB2-negative cohorts (9.1 weeks and 7.6 weeks, respectively). Median survival was 29.4 weeks in ERBB2-positive patients and 18.6 weeks in ERBB2-negative patients.

Two open-label Phase II trials that evaluated the safety and efficacy of lapatinib monotherapy in patients with trastuzumab-refractory, ERBB2-positive advanced or metastatic breast cancer found similar results. Blackwell et al evaluated 78 patients with ERBB2-positive metastatic breast cancer refractory to trastuzumab and found a clinical benefit rate (complete response, partial response + stable disease $\geq 16$ weeks) of $21.8 \%$ and a partial response rate of $7.7 \% .{ }^{57}$ Patients were treated with either $1250 \mathrm{mg}$ or $1500 \mathrm{mg}$ daily after protocol amendment. The study concluded that lapatinib as a single agent has clinical activity associated with manageable toxic effects, the most common being rash (47\%), diarrhea (46\%), and nausea (31\%). Another openlabel Phase II trial conducted in Japan of 45 patients with ERBB2-positive metastatic breast cancer refractory to previous anthracycline, taxane, capecitabine, and trastuzumab therapy showed a clinical benefit rate (complete response, partial response, + stable disease $\geq 24$ weeks) of $33.3 \%{ }^{58}$

Gomez et al reported the results of a randomized, openlabel Phase II study of lapatinib as first-line monotherapy for ERBB2-positive advanced or metastatic breast cancer. ${ }^{59}$ Patients were randomly assigned to one of two dose groups and received either $1500 \mathrm{mg}$ once daily or $500 \mathrm{mg}$ twice daily. Seventy-six percent of the patients had both estrogen receptor- and progesterone receptor-negative tumors. Overall, there was a $24 \%$ response rate, clinical benefit rate (complete response, partial response, + stable disease $\geq 24$ weeks) of $31 \%$, progression-free survival at six months, and no significant difference in outcomes between the two groups.

\section{Lapatinib-chemotherapy combinations}

A 2006 study that compared lapatinib plus capecitabine with capecitabine alone established that the combination therapeutic regimen is superior to capecitabine monotherapy in women with ERBB2-positive advanced breast cancer whose disease has progressed after anthracycline, taxane, or trastuzumab treatments. ${ }^{34}$ The primary endpoint was time to tumor progression (hazard ratio $[\mathrm{HR}]=0.49 ; 95 \%$ confidence interval [CI]: $0.34-0.71 ; P<0.001)$. Three hundred twentyfour women were randomly assigned to receive oral lapatinib $1250 \mathrm{mg}$ daily plus capecitabine $2000 \mathrm{mg} / \mathrm{m}^{2}$ given in two divided doses on days 1-14 of a 21-day cycle, or capecitabine monotherapy at $2500 \mathrm{mg} / \mathrm{m}^{2}$ given in two divided doses on days 1-14 of a 21-day cycle. The results showed an increased median time to tumor progression for patients in the combination arm compared with those on the monotherapy regimen (8.4 months versus 4.4 months, respectively). Moreover, these results were achieved without compromising cardiac function or increasing toxicity.

Another multicenter, randomized, double-blind, placebocontrolled Phase III study comparing lapatinib plus paclitaxel versus paclitaxel alone for first-line treatment in 580 women with metastatic disease was reported in $2007 .{ }^{60}$ Patients were randomized into two groups where one received oral lapatinib $1500 \mathrm{mg}$ daily plus paclitaxel $175 \mathrm{mg} / \mathrm{m}^{2}$ every three weeks and the other group received placebo plus paclitaxel $175 \mathrm{mg} / \mathrm{m}^{2}$ every three weeks. In an intent-to-treat analysis of investigators' assessments, the ERBB2 untested 
or unknown population that received combination therapy had a significantly higher response rate when compared with paclitaxel monotherapy (35.1\% versus $25.3 \%$, respectively; $P=0.008)$. The clinical benefit rate at six months was also significantly higher with combination therapy than with paclitaxel monotherapy $(40.5 \%$ versus $31.9 \%$; $P=0.025)$. However, no significant differences in the median duration of response, time to tumor progression, or overall survival were observed. When the results were evaluated by ERBB2-status subset analysis, patients with ERBB2 amplification who received combination therapy had significant improvement in response rate compared with the paclitaxel monotherapy regimen ( $60 \%$ versus $36 \% ; P=0.027)$ and improved median time to tumor progression (6.1 versus 5.8 months; $P=0.011$ ). In the ERBB2-untested population, combination therapy showed no improvement over monotherapy (time to tumor progression 6.7 versus 5.3 months, respectively).

\section{Lapatinib-hormonal therapy combinations}

Resistance to endocrine therapy poses a challenge in the treatment of hormone receptor-positive metastatic breast cancers. Cross-talk between pathways involving ERBB receptors and hormone receptors appears to contribute to endocrine therapy resistance. ${ }^{61-65}$ ERBB2-overexpression confers resistance to established endocrine therapies, and patients with hormone receptor-positive and ERBB2-positive metastatic breast cancers who were treated with trastuzumab combined with the aromatase inhibitor, anastrozole (Arimidex ${ }^{\circledR}$; AstraZeneca, London, UK), had twice the median progression-free survival when compared with those treated with anastrozole alone (2.4 versus 4.8 months, respectively). ${ }^{62,66-69}$ Consequently, to overcome endocrine resistance, therapies combining ERBB2 pathway inhibitors with endocrine manipulation have been studied.

Preclinical studies evaluated the potential of lapatinib in combination with estrogen receptor blockade by tamoxifen (Nolvadex ${ }^{\circledR}$; AstraZeneca) to restore responsiveness to tamoxifen-resistant breast cancers in culture and in xenograft tumors in mice. Chu et al reported that combined treatment with tamoxifen and lapatinib caused a greater increase in the cell cycle inhibitor, p27, and in p27-mediated cell cycle arrest in $\mathrm{G}_{1}$ phase than was observed with either drug alone. ${ }^{2}$ In breast cancer xenograft studies, tumors that were originally tamoxifen-stimulated showed inhibition by combined lapatinib plus tamoxifen.

In 2009, a Phase III study was reported that evaluated addition of lapatinib to the aromatase inhibitor, letrozole (Femara ${ }^{\circledR}$; Novartis International AG), as a first-line treatment of hormone receptor-positive metastatic breast cancer in postmenopausal women $(\mathrm{n}=1286) .{ }^{70}$ Patients were randomized to receive daily treatment with $2.5 \mathrm{mg}$ of letrozole plus $1500 \mathrm{mg}$ of lapatinib or $2.5 \mathrm{mg}$ letrozole plus placebo. While addition of lapatinib to letrozole therapy did not provide additional benefit in this postmenopausal patient group overall, the subset analysis of ERBB2-positive cancers was instructive. Two hundred nineteen of the 1286 patients enrolled had ERBB2-overexpressing tumors. Using progression-free survival as the primary endpoint in the ERBB2-overexpressing cancers, the analysis revealed that the addition of lapatinib to letrozole significantly lowered risk of disease progression compared with letrozole alone $(\mathrm{HR}=0.71 ; 95 \% \mathrm{CI}$ : $0.53-0.96 ; P=0.019)$ where the progression-free survival was 8.2 months versus 3.0 months, respectively. The objective response rate $(28 \%$ versus $15 \%$; odds ratio [OR], 0.4 ; 95\% CI: $0.2-0.9 ; P=0.21))$ and clinical benefit rate $(48 \%$ versus $29 \%$; OR, 0.4 ; 95\% CI: $0.2-0.8 ; P=0.003$ ) were also significantly greater in the lapatinib plus letrozole-treated ERBB2-overexpressing cancers. On the other hand, patients whose cancers were ERBB2-negative $(n=952)$ did not show improvement in progression-free survival. As of February 2010, lapatinib plus letrozole is now an FDA-approved regimen for hormone receptor-positive and ERBB2-overexpressing postmenopausal metastatic breast cancers. ${ }^{71}$

\section{Lapatinib-trastuzumab combinations}

Konecny et al investigated the therapeutic potential of lapatinib by examining its effect on the growth of 31 trastuzumabresistant ERRB-2 positive human breast cancer cell lines. ${ }^{50}$ Concentration-dependent antiproliferative effects of lapatinib were seen in all cell lines tested. However, the extent of growth inhibition by lapatinib varied widely between individual cell lines and was significantly correlated with level of ERBB2 expression. ERBB2 gene amplification and ERBB2 protein overexpression were associated with a higher sensitivity to lapatinib in vitro across the tested cell lines. Significant reduction in the volume of human breast cancer xenografts in athymic mice was observed with lapatinib when compared with untreated controls. Synergistic drug interactions between lapatinib and trastuzumab were also seen in four different ERBB2-overexpressing cell lines. This study, reported in 2006, provided the biologic rationale to test lapatinib as a single agent or in combination with trastuzumab in ERBB2-overexpressing patients with clinical resistance to trastuzumab.

Clinical trials of lapatinib in conjunction with trastuzumab have tested the potential superiority of this combination 
over lapatinib monotherapy for trastuzumab-resistant metastatic breast cancer. The activity of lapatinib in combination with trastuzumab in patients with advanced, ERBB2-positive, trastuzumab-refractory metastatic breast cancer was reported in 2008. This Phase I study evaluated this regimen's safety, optimally tolerated regimen, and pharmacokinetics. ${ }^{72}$ Cohorts of three patients were treated with escalating doses of lapatinib from $750 \mathrm{mg}$ to $1500 \mathrm{mg}$ administered once daily in combination with trastuzumab ( $4 \mathrm{mg} / \mathrm{kg}$ loading dose, then $2 \mathrm{mg} / \mathrm{kg}$ weekly). Additional patients were enrolled to evaluate the pharmacokinetics of both agents alone and in combination, once the optimally tolerated regimen was determined. The dose-limiting toxicities of a sequence of dose levels were studied. Based on the two dose-limiting toxicities reported at $1250 \mathrm{mg}$ lapatinib with trastuzumab, and the higher frequency of fatigue impacting tolerability, the combination dose of lapatinib $1000 \mathrm{mg}$ and with standard weekly trastuzumab was selected as the optimally tolerated regimen.

A subsequent Phase II study evaluated the coadministration of trastuzumab and lapatinib versus lapatinib alone in metastatic ERBB2-positive disease refractory to trastuzumab therapy and showed that patients receiving combined treatment had a significantly improved median progression-free survival (12 months versus 8.1 months), a 27\% reduction in risk of disease progression $(P=0.008)$, an increased clinical benefit rate $(24.7 \%$ versus $12.4 \%)$, a greater tumor response rate $(10.3 \%$ versus $6.9 \%)$ and a trend toward improved survival. ${ }^{73}$ An updated survival analysis, reported by Blackwell et al in $2009,{ }^{74}$ concluded that combination therapy with lapatinib plus trastuzumab was superior to lapatinib alone. The median overall survival of 60.7 weeks for combination therapy was significantly higher than the overall survival of 41.4 weeks for lapatinib alone (HR 0.74; 95\% CI: 0.57-0.97; $P=0.026)$. Further, the survival benefit was maintained upon adjusting for baseline prognostic factors (HR $0.71 ; 95 \%$ CI: $0.54-0.93 ; P=0.012) .{ }^{74}$ Further clinical trials evaluating trastuzumab-lapatinib combination therapy are currently underway in metastatic and early breast cancer.

\section{Lapatinib and inflammatory breast cancer}

Although the incidence of inflammatory breast cancer is low ( $<5 \%$ of breast cancers) in the US, this form of breast cancer is very aggressive, affects a younger population, and has a low overall survival. Inflammatory breast cancer is characterized by rapid onset of swelling and erythema of the breast, and often does not present with a palpable breast mass. ${ }^{75}$ Dermal lymphatic invasion is characteristic of this disease which often shows overexpression of ERBB2. Lapatinib has exhibited encouraging activity in inflammatory breast cancer. ${ }^{76}$ A Phase I trial showed four clinical responses (complete response/partial response) in five patients with inflammatory breast cancer treated with lapatinib and led to Phase II studies that are continuing the evaluation of lapatinib in inflammatory breast cancer. ${ }^{77}$

Johnston et al conducted a Phase II study in patients with recurrent or anthracycline refractory inflammatory breast cancer who had not received previous trastuzumab therapy. ${ }^{78}$ The 45 enrolled patients were grouped into two cohorts based on their ERBB2 and ERBB1 profiles (cohort A: ERBB2-positive; cohort B: ERBB2-negative and ERBB1positive). Fifty percent of cohort A (15 of 30 patients) exhibited a clinical response, while only $6.7 \%$ (one of 15 patients) in cohort B had a clinical response. Tumor markers predictive of lapatinib response in cohort A included phosphorylated ERBB3 expression $(P=0.021)$, phosphorylated ERBB2/ERBB3 coexpression $(P=0.005)$, and absence of p53 expression $(P=0.033)$.

Another Phase II trial reported by Spector et al, which evaluated lapatinib monotherapy for treatment-refractory inflammatory breast cancer, indicated that high ERBB2, phosphorylated-ERBB2, and IGF-IR coexpression are predictors for clinical response to lapatinib monotherapy in patients with treatment-resistant inflammatory breast cancer. ${ }^{79}$ ERBB2 overexpression, but not ERBB1 expression alone, indicates sensitivity to lapatinib in inflammatory breast cancer. Seventeen patients were evaluated from the 34 patients enrolled. Cohort A was comprised of ERBB2positive patients, irrespective of ERBB1 status, while cohort B consisted of patients with ERBB1-positive and ERBB2negative tumors. Cohort A showed clinical responses in eight of the 11 patients, whereas none of the six patients in cohort B showed any clinical response.

Expanding on these findings, Kaufman et al reported on an open-label Phase II study of $1500 \mathrm{mg}$ of lapatinib given once daily in 126 patients with relapsed or refractory ERBB2-positive inflammatory breast cancer. ${ }^{80}$ Although none of the patients had a complete response, $39 \%$ of the patients had an overall response. The findings included a median response of 19.4 months (95\% CI: 12.8-26.4) and a median overall survival of 11.2 months (95\% CI: 9.1-13.5) for patients who responded to lapatinib, compared with a median overall survival of 8.4 months (95\% CI: 5.9-9.4) for nonresponders to lapatinib.

An open-label Phase II study in 23 patients with newly diagnosed inflammatory breast cancer treated with lapatinib 
in combination with paclitaxel as neoadjuvant therapy showed a clinical response in 95\% (20/21) patients with ERBB2positive tumors and in two of two patients with ERBB1positive/ERBB2-negative tumors. ${ }^{81}$ These encouraging results support further clinical investigation of combination regimens containing lapatinib prior to surgery for inflammatory breast cancer patients.

\section{Treatment of central nervous system metastasis}

It has been estimated that $15 \%$ of advanced breast cancer patients develop symptomatic brain metastases. ${ }^{82,83}$ A metaanalysis of the randomized controlled trials evaluating trastuzumab treatment in early breast cancer found that development of brain metastasis was significantly higher for trastuzumab-treated patients $(P=0.033)$, but is overall low-risk $(0.62 \%) .{ }^{84}$ Unfortunately, other than treatment with steroids, radiotherapy or surgery, no treatment currently provides long-term efficacy for brain metastases.$^{85} \mathrm{It}$ has been postulated that due to its small size, lapatinib may circumvent this problem by permeating the central nervous system to prevent or reduce brain metastases.

In a 2008 open-label Phase II trial, Lin et al reported early evidence for a clinical benefit when ERBB2-positive breast cancer patients with new or progressive brain metastases were given lapatinib monotherapy at a starting dose of $750 \mathrm{mg}$ twice a day in four-week cycles. ${ }^{86}$ Thirty-nine patients who had developed brain metastases while receiving trastuzumab were evaluated. Eligibility requirements included prior trastuzumab and documented central nervous system progression after radiation therapy. If patients had not received previous radiation therapy, the patients were required to be asymptomatic. The median time to tumor progression was three months (95\% CI: 2.3-3.7) and 11.3 months for the one patient with a central nervous system objective response. Seven patients were progression-free in both central nervous system and noncentral nervous system sites at 16 weeks, and three patients had a decline in volume of central nervous system metastasis of $>30 \%$.

Since both capecitabine and lapatinib have potential efficacy for central nervous system disease, these initial data with lapatinib monotherapy prompted Geyer et al to suggest that combination lapatinib and capecitabine therapy may delay progression or even prevent the onset of central nervous system metastasis. ${ }^{34}$ Data from trials comparing trastuzumab and lapatinib in the adjuvant and neoadjuvant settings should yield information on their respective abilities to reduce subsequent brain metastasis and are eagerly awaited.

\section{Administration}

The following presents administration guidelines for FDAapproved uses of lapatinib. Lapatinib is administered in $250 \mathrm{mg}$ tablets in combination with capecitabine over 21-day cycles. ${ }^{54}$ The recommended dosage of lapatinib for patients with advanced or metastatic breast cancer who are positive for ERBB2 and have previously received therapy including an anthracycline, a taxane, and trastuzumab, is $1250 \mathrm{mg}$ given once daily on days 1-21 continuously in combination with capecitabine at $2000 \mathrm{mg} / \mathrm{m}^{2} /$ day (administered orally two times a day) on days 1-14. While whole tablets of lapatinib should be administered on an empty stomach, one hour before or after a meal, capecitabine should be taken on a full stomach. ${ }^{33,87}$ As stated previously, since lapatinib is an inhibitor of CYP3A4, if coadministration of a CYP3A4 inhibitor is necessary, a dose reduction of lapatinib to $500 \mathrm{mg}$ /day may be considered. Additionally, due to gastric $\mathrm{pH}$ fluctuations, antacids should be avoided for one hour before and after lapatinib administration. ${ }^{33}$ Treatment should be continued until disease progression or the onset of intolerable adverse effects.

As of 2010, lapatinib is indicated for use in combination with letrozole for the treatment of postmenopausal women with hormone receptor-positive metastatic breast cancer with ERBB2 overexpression. ${ }^{33}$ In combination with letrozole, the recommended dosage of lapatinib is $1500 \mathrm{mg}$ given once daily continuously in combination with letrozole. When coadministered with lapatinib, the recommended dose of letrozole is $2.5 \mathrm{mg}$ once daily.

Patients should be monitored for cardiac, pulmonary, and hepatic function while on a lapatinib regimen. ${ }^{33}$ Left ventricular ejection fraction should be evaluated in all patients prior to the initiation of treatment with lapatinib as a baseline, using means such as echocardiography or multiple-gated acquisition scans. Pulmonary symptoms indicative of interstitial lung disease or pneumonitis should also be monitored in all patients. Patients' liver function tests, including transaminases, bilirubin, and alkaline phosphatase, should also be examined prior to treatment and every 4-6 weeks during treatment. Further, because myelosuppression is an adverse effect of capecitabine, complete blood counts should be performed according to the treating physician's judgment.

\section{Adverse effects and tolerability}

Studies have shown lapatinib to be a generally well tolerated drug. The most common clinical toxicities related to lapatinib monotherapy administered at $1500 \mathrm{mg}$ once daily in the Phase II study conducted by Burstein et al were diarrhea (54\%), rash (30\%), nausea (24\%), vomiting (14\%), fatigue 
(14\%), and anorexia (10\%). ${ }^{56}$ These adverse effects were mostly Grade 1 or 2 in severity, with a maximum severity of Grade 3 by National Cancer Institute Common Terminology Criteria for Adverse Events.

When used in combination regimens, lapatinib-related adverse effects are increased. Geyer et al reported the most common adverse events from lapatinib plus capecitabine therapy were diarrhea $(60 \%)$, palmoplantar erythrodysesthesia (hand-foot) syndrome (49\%), nausea (44\%), rash (27\%), vomiting $(26 \%)$, and fatigue (18\%), with most events of Grade 1, 2, or 3 severity. ${ }^{34}$ Two of 163 (1\%) women who received combination therapy with capecitabine experienced Grade 4 diarrhea. When lapatinib was combined with letrozole, the most common adverse reactions were diarrhea $(64 \%)$, rash $(44 \%)$, nausea $(31 \%)$, fatigue $(20 \%)$, and arthralgia (19\%), with the majority being Grade 1 or $2 .{ }^{70}$ Sixty patients $(10 \%)$ who received lapatinib plus letrozole combination therapy had Grade 3 or 4 diarrhea.

Lapatinib-associated cardiac toxicity has been reported at a low frequency. Cardiac toxicity was an unexpected adverse event found from early preclinical trials of trastuzumab metastatic breast cancer patients. Because cardiac toxicity was a serious toxicity in the pivotal Phase III trial of trastuzumab in the treatment of metastatic breast cancer, there was initial concern about the potential cardiotoxicity of lapatinib. ${ }^{22}$ However, Burstein et al reported only one patient who had a Grade 3 decrease in left ventricular ejection fraction. ${ }^{56}$ Lin et al reported no cases of symptomatic congestive heart failure, although four patients who had received a median of two previous trastuzumab-containing chemotherapy regimens had asymptomatic decreases in left ventricular ejection fraction to $<50 \% .{ }^{86} \mathrm{~A}$ pooled analysis of trials involving over 12,000 patients revealed that cardiac toxicity was observed in only $0.4-4 \%$ of patients. ${ }^{23,24,88,89}$ A retrospective analysis of lapatinib-associated cardiac toxicity was conducted by Perez et al in 3689 lapatinibtreated patients. ${ }^{90}$ Data from 44 Phase I-III clinical studies of lapatinib monotherapy and combination therapy showed low levels of cardiotoxicity for lapatinib. Cardiac events were reported in 60 patients $(1.6 \%)$. These patients had previously been treated with anthracyclines $(n=12)$, trastuzumab $(n=14)$, or neither $(n=34)$. Overall, no cardiac mortality was recorded. The study concluded that cardiac events were usually asymptomatic, caused reversible decreases in left ventricular ejection fraction, and occurred at similar rates in patients who had and had not received previous treatments with anthracyclines and trastuzumab. Although the long-term cardiac effects of lapatinib are not known at present, re-evaluation of longterm follow-up data will ultimately be forthcoming.

\section{Cost considerations}

The wholesale price of lapatinib in the US is on average $\$ 26$ per $250 \mathrm{mg}$ tablet. At the recommended $1250 \mathrm{mg} /$ day dosage, the therapy currently costs approximately $\$ 130$ per day or $\$ 2800$ for each 21 -day cycle. ${ }^{91}$ To assess the total cost of lapatinib treatment, combination agents such as capecitabine and letrozole need to be included. The costbenefit analysis for this agent is a serious consideration for developing nations and for countries with limited resources for health care delivery.

\section{Conclusion}

A myriad of targeted therapies have emerged that show activity against ERBB2-positive breast cancers. The use of lapatinib as monotherapy or in combination with conventional cancer treatments is currently being assessed in various clinical trials. Although lapatinib is a small molecule tyrosine kinase inhibitor of both ERBB1 and ERBB2, its action appears to be limited to ERBB2-positive disease. It has been approved for use in combination with capecitabine in the treatment of ERBB2-positive metastatic breast cancer patients whose disease has progressed after trastuzumab therapy and in combination with letrozole for the treatment of postmenopausal women with ERBB2-positive and hormone receptor-positive metastatic breast cancer. Lapatinib monotherapy is generally well tolerated and is not associated with cardiotoxicity.

Further trials evaluating lapatinib are currently ongoing. Two large world-wide randomized studies, ALTTO and Neo-ALTTO (Adjuvant Lapatinib and/or Trastuzumab Treatment Optimization), will investigate lapatinib alone or in combination with trastuzumab in the adjuvant and neoadjuvant settings, respectively, in patients with early ERBB2-positive breast cancer. These international, randomized, open-label Phase III trials intend to enroll 8000 patients with ERBB2positive breast cancers. Other trials, including the investigation of lapatinib with other chemotherapeutic agents, such as vinorelbine (Navelbine ${ }^{\circledR}$; GlaxoSmithKline), and lapatinib in combination with letrozole in the neoadjuvant setting, are also in development.

\section{Disclosure}

The authors report no conflicts of interest in this work. 


\section{References}

1. Jemal A, Siegel R, Ward E, Hao Y, Xu J, Thun MJ. Cancer statistics, 2009. CA Cancer J Clin. 2009;59(4):225-249.

2. Chu I, Blackwell K, Chen S, Slingerland J. The dual ErbB1/ErbB2 inhibitor, lapatinib (GW572016), cooperates with tamoxifen to inhibit both cell proliferation- and estrogen-dependent gene expression in antiestrogen-resistant breast cancer. Cancer Res. 2005;65(1):18-25.

3. Tsutsui S, Ohno S, Murakami S, Hachitanda Y, Oda S. Prognostic value of epidermal growth factor receptor (EGFR) and its relationship to the estrogen receptor status in 1029 patients with breast cancer. Breast Cancer Res Treat. 2002;71(1):67-75.

4. Nicholson S, Wright C, Sainsbury JR, et al. Epidermal growth factor receptor (EGFr) as a marker for poor prognosis in node-negative breast cancer patients: Neu and tamoxifen failure. J Steroid Biochem Mol Biol. 1990;37(6):811-814.

5. Agrawal A, Gutteridge E, Gee JM, Nicholson RI, Robertson JF. Overview of tyrosine kinase inhibitors in clinical breast cancer. Endocr Relat Cancer. 2005;12 Suppl 1:S135-S144.

6. Rusch V, Baselga J, Cordoncardo C, et al. Differential expression of the epidermal growth-factor receptor and its ligands in primary nonsmall cell lung cancers and adjacent benign lung. Cancer Res. 1993;53(10):2379-2385.

7. Selvaggi G, Novello S, Torri V, et al. Epidermal growth factor receptor overexpression correlates with a poor prognosis in completely resected non-small-cell lung cancer. Ann Oncol. 2004;15(1):28-32.

8. Onn A, Correa AM, Gilcrease M, et al. Synchronous overexpression of epidermal growth factor receptor and HER2-neu protein is a predictor of poor outcome in patients with stage I non-small cell lung cancer. Clin Cancer Res. 2004;10(1 Pt 1):136-143.

9. Ohtsuka K, Ohnishi H, Furuyashiki G, et al. Clinico-pathological and biological significance of tyrosine kinase domain gene mutations and overexpression of epidermal growth factor receptor for lung adenocarcinoma. J Thorac Oncol. 2006;1(8):787-795.

10. Baselga J, Swain SM. Novel anticancer targets: Revisiting ERBB2 and discovering ERBB3. Nat Rev Cancer. 2009;9(7):463-475.

11. Prickett TD, Agrawal NS, Wei X, et al. Analysis of the tyrosine kinome in melanoma reveals recurrent mutations in ERBB4. Nat Genet. 2009; 41(10):1127-1132.

12. Gadella TW Jr, Jovin TM. Oligomerization of epidermal growth factor receptors on A431 cells studied by time-resolved fluorescence imaging microscopy. A stereochemical model for tyrosine kinase receptor activation. J Cell Biol. 1995;129(6):1543-1558.

13. Moriki T, Maruyama H, Maruyama IN. Activation of preformed EGF receptor dimers by ligand-induced rotation of the transmembrane domain. J Mol Biol. 2001;311(5):1011-1026.

14. Schoeberl B, Faber AC, Li D, et al. An ErbB3 antibody, MM-121, is active in cancers with ligand-dependent activation. Cancer Res. 2010;70(6):2485-2494.

15. Yarden Y, Sliwkowski MX. Untangling the ErbB signalling network. Nat Rev Mol Cell Biol. 2001;2(2):127-137.

16. Press MF, Slamon DJ, Flom KJ, Park J, Zhou JY, Bernstein L. Evaluation of HER-2/neu gene amplification and overexpression: Comparison of frequently used assay methods in a molecularly characterized cohort of breast cancer specimens. J Clin Oncol. 2002;20(14):3095-3105.

17. Wolff AC, Hammond ME, Schwartz JN, et al. American Society of Clinical Oncology/College of American Pathologists guideline recommendations for human epidermal growth factor receptor 2 testing in breast cancer. Arch Pathol Lab Med. 2007;131(1):18-43.

18. Sauter G, Lee J, Bartlett JM, Slamon DJ, Press MF. Guidelines for human epidermal growth factor receptor 2 testing: Biologic and methodologic considerations. J Clin Oncol. 2009;27(8):1323-1333.

19. Spector NL, Blackwell KL. Understanding the mechanisms behind trastuzumab therapy for human epidermal growth factor receptor 2-positive breast cancer. J Clin Oncol. 2009;27(34):5838-5847.

20. Pegram M, Hsu S, Lewis G, et al. Inhibitory effects of combinations of HER-2/neu antibody and chemotherapeutic agents used for treatment of human breast cancers. Oncogene. 1999;18(13):2241-2251.
21. Vogel CL, Cobleigh MA, Tripathy D, et al. Efficacy and safety of trastuzumab as a single agent in first-line treatment of HER2-overexpressing metastatic breast cancer. J Clin Oncol. 2002;20(3):719-726.

22. Slamon DJ, Leyland-Jones B, Shak S, et al. Use of chemotherapy plus a monoclonal antibody against HER2 for metastatic breast cancer that overexpresses HER2. N Engl J Med. 2001;344(11):783-792.

23. Piccart-Gebhart MJ, Procter M, Leyland-Jones B, et al. Trastuzumab after adjuvant chemotherapy in HER2-positive breast cancer. $N$ Engl J Med. 2005;353(16):1659-1672.

24. Romond EH, Perez EA, Bryant J, et al. Trastuzumab plus adjuvant chemotherapy for operable HER2-positive breast cancer. $N$ Engl J Med. 2005;353(16):1673-1684.

25. Lan $\mathrm{KH}, \mathrm{Lu} \mathrm{CH}, \mathrm{Yu} \mathrm{D}$. Mechanisms of trastuzumab resistance and their clinical implications. Ann NY Acad Sci. 2005;1059:70-75.

26. Faneyte IF, Peterse JL, van TH, et al. Predicting early failure after adjuvant chemotherapy in high-risk breast cancer patients with extensive lymph node involvement. Clin Cancer Res. 2004;10(13):4457-4463.

27. Montemurro F, Donadio M, Clavarezza M, et al. Outcome of patients with HER2-positive advanced breast cancer progressing during trastuzumab-based therapy. Oncologist. 2006;11(4):318-324.

28. Lewis Phillips GD, Li G, Dugger DL, et al. Targeting HER2-positive breast cancer with trastuzumab-DM1, an antibody-cytotoxic drug conjugate. Cancer Res. 2008;68(22):9280-9290.

29. Agus DB, Gordon MS, Taylor C, et al. Phase I clinical study of pertuzumab, a novel HER dimerization inhibitor, in patients with advanced cancer. J Clin Oncol. 2005;23(11):2534-2543.

30. Gianni L, Llado A, Bianchi G, et al. Open-label, phase II, multicenter, randomized study of the efficacy and safety of two dose levels of pertuzumab, a human epidermal growth factor receptor 2 dimerization inhibitor, in patients with human epidermal growth factor receptor 2-negative metastatic breast cancer. J Clin Oncol. 2010;28(7):1131-1137.

31. Gelmon KA, Furnoleau P, Verma S, et al. Results of a Phase II trial of trastuzumab $(\mathrm{H})$ and pertuzumab $(\mathrm{P})$ in patients (pts) with HER2positive metastatic breast cancer (MBC) who had progressed during trastuzumab therapy. J Clin Oncol. 2008;26(47S):1026.

32. Citri A, Yarden Y. EGF-ERBB signalling: Towards the systems level. Nat Rev Mol Cell Biol. 2006;7(7):505-516.

33. Tykerb ${ }^{\circledR}$ (Lapatinib, Package insert). Research Triangle Park, NC; GlaxoSmithKline: 2010

34. Geyer CE, Forster J, Lindquist D, et al. Lapatinib plus capecitabine for HER2-positive advanced breast cancer. $N$ Engl J Med. 2006; 355(26):2733-2743.

35. Spector NL, Xia W, Burris H III, et al. Study of the biologic effects of lapatinib, a reversible inhibitor of ErbB1 and ErbB2 tyrosine kinases, on tumor growth and survival pathways in patients with advanced malignancies. J Clin Oncol. 2005;23(11):2502-2512.

36. Lackey KE. Lessons from the drug discovery of lapatinib, a dual ErbB1/2 tyrosine kinase inhibitor. Curr Top Med Chem. 2006;6(5): 435-460.

37. Wood ER, Truesdale AT, McDonald OB, et al. A unique structure for epidermal growth factor receptor bound to GW572016 (lapatinib): Relationships among protein conformation, inhibitor off-rate, and receptor activity in tumor cells. Cancer Res. 2004;64(18):6652-6659.

38. Anido J, Scaltriti M, Bech Serra JJ, et al. Biosynthesis of tumorigenic HER2 C-terminal fragments by alternative initiation of translation. EMBO J. 2006;25(13):3234-3244.

39. Pupa SM, Menard S, Morelli D, Pozzi B, De PG, Colnaghi MI. The extracellular domain of the c-erbB-2 oncoprotein is released from tumor cells by proteolytic cleavage. Oncogene. 1993;8(11):2917-2923.

40. Scaltriti M, Rojo F, Ocana A, et al. Expression of p95HER2, a truncated form of the HER2 receptor, and response to anti-HER2 therapies in breast cancer. J Natl Cancer Inst. 2007;99(8):628-638.

41. Christianson TA, Doherty JK, Lin YJ, et al. NH2-terminally truncated HER-2/neu protein: Relationship with shedding of the extracellular domain and with prognostic factors in breast cancer. Cancer Res. 1998;58(22):5123-5129. 
42. Molina MA, Saez R, Ramsey EE, et al. NH(2)-terminal truncated HER-2 protein but not full-length receptor is associated with nodal metastasis in human breast cancer. Clin Cancer Res. 2002;8(2): 347-353.

43. Bramwell VH, Doig GS, Tuck AB, et al. Changes over time of extracellular domain of HER2 (ECD/HER2) serum levels have prognostic value in metastatic breast cancer. Breast Cancer Res Treat. 2009;114(3): 503-511.

44. Ali SM, Carney WP, Esteva FJ, et al. Serum HER-2/neu and relative resistance to trastuzumab-based therapy in patients with metastatic breast cancer. Cancer. 2008;113(6):1294-1301.

45. Nunes RA, Harris LN. The HER2 extracellular domain as a prognostic and predictive factor in breast cancer. Clin Breast Cancer. 2002;3(2): $125-135$.

46. Mazouni C, Hall A, Broglio K, et al. Kinetics of serum HER-2/neu changes in patients with HER-2-positive primary breast cancer after initiation of primary chemotherapy. Cancer. 2007;109(3):496-501.

47. Sperinde J, Jin X, Banerjee J, et al. Quantitation of p95HER2 in paraffin sections by using a p 95 -specific antibody and correlation with outcome in a cohort of trastuzumab-treated breast cancer patients. Clin Cancer Res. 2010;16(16):4226-4235.

48. Saez R, Molina MA, Ramsey EE, et al. p95HER-2 predicts worse outcome in patients with HER-2-positive breast cancer. Clin Cancer Res. 2006;12(2):424-431.

49. Rusnak DW, Lackey K, Affleck K, et al. The effects of the novel, reversible epidermal growth factor receptor/ErbB-2 tyrosine kinase inhibitor, GW2016, on the growth of human normal and tumor-derived cell lines in vitro and in vivo. Mol Cancer Ther. 2001;1(2):85-94.

50. Konecny GE, Pegram MD, Venkatesan N, et al. Activity of the dual kinase inhibitor lapatinib (GW572016) against HER-2-overexpressing and trastuzumab-treated breast cancer cells. Cancer Res. 2006;66(3): $1630-1639$

51. Bence AK, Anderson EB, Halepota MA, et al. Phase I pharmacokinetic studies evaluating single and multiple doses of oral GW572016, a dual EGFR-ErbB2 inhibitor, in healthy subjects. Invest New Drugs. 2005;23(1):39-49.

52. Burris HA III, Hurwitz HI, Dees EC, et al. Phase I safety, pharmacokinetics, and clinical activity study of lapatinib (GW572016), a reversible dual inhibitor of epidermal growth factor receptor tyrosine kinases, in heavily pretreated patients with metastatic carcinomas. J Clin Oncol. 2005;23(23):5305-5313.

53. Food and Drug Administration. Approval package for Application number NDA 22-059. 2010.

54. Medina PJ, Goodin S. Lapatinib: A dual inhibitor of human epidermal growth factor receptor tyrosine kinases. Clin Ther. 2008;30(8): $1426-1447$.

55. Moy B, Goss PE. Lapatinib-associated toxicity and practical management recommendations. Oncologist. 2007;12(7):756-765.

56. Burstein HJ, Storniolo AM, Franco S, et al. A phase II study of lapatinib monotherapy in chemotherapy-refractory HER2-positive and HER2-negative advanced or metastatic breast cancer. Ann Oncol. 2008;19(6):1068-1074.

57. Blackwell KL, Burstein HJ, Pegram M, et al. Determining relevant biomarkers from tissue and serum that may predict response to single agent lapatinib in trastuzumab refractory metastatic breast cancer. $J$ Clin Oncol. 2005;23 Suppl 16:193s.

58. Iwata H, Toi M, Fujiwara Y. Phase II clinical study of lapatinib (GW572016) in patients with advanced or metastatic breast cancer. Presented at: San Antonio Breast Cancer Symposium, San Antonio, TX. Dec 14-17, 2006.

59. Gomez HL, Doval DC, Chavez MA, et al. Efficacy and safety of lapatinib as first-line therapy for ErbB2-amplified locally advanced or metastatic breast cancer. J Clin Oncol. 2008;26(18):2999-3005.

60. di Leo A, Gomez H, Aziz Z, et al. Lapatinib (L) with paclitaxel compared to paclitaxel as first-line treatment for patients with metastatic breast cancer: A phase III randomized, double-blind study of 580 patients. J Clin Oncol. 2007;25 Suppl:34s.
61. Nicholson RI, Gee JM, Harper ME. EGFR and cancer prognosis. Eur J Cancer. 2001;37 Suppl 4:S9-S15.

62. Lipton A, Ali SM, Leitzel K, et al. Serum HER-2/neu and response to the aromatase inhibitor letrozole versus tamoxifen. J Clin Oncol. 2003;21(10):1967-1972.

63. Arpino G, Wiechmann L, Osborne CK, Schiff R. Crosstalk between the estrogen receptor and the HER tyrosine kinase receptor family: Molecular mechanism and clinical implications for endocrine therapy resistance. Endocr Rev. 2008;29(2):217-233.

64. Hurtado A, Holmes KA, Geistlinger TR, et al. Regulation of ERBB2 by oestrogen receptor-PAX2 determines response to tamoxifen. Nature. 2008;456(7222):663-666

65. Yang Z, Barnes CJ, Kumar R. Human epidermal growth factor receptor 2 status modulates subcellular localization of and interaction with estrogen receptor alpha in breast cancer cells. Clin Cancer Res. 2004;10(11): 3621-3628.

66. Benz CC, Scott GK, Sarup JC, et al. Estrogen-dependent, tamoxifenresistant tumorigenic growth of MCF-7 cells transfected with HER2/ neu. Breast Cancer Res Treat. 1992;24(2):85-95.

67. Pietras RJ, Arboleda J, Reese DM, et al. HER-2 tyrosine kinase pathway targets estrogen receptor and promotes hormone-independent growth in human breast cancer cells. Oncogene. 1995;10(12):2435-2446.

68. Shin I, Miller T, Arteaga CL. ErbB receptor signaling and therapeutic resistance to aromatase inhibitors. Clin Cancer Res. 2006;12(3 Pt 2): S1008-S1012.

69. Mackey JR, Kaufman B, Clemens M. Trastuzumab prolongs progression-free survival in hormone-dependent and HER2-positive metastatic breast cancer. Breast Cancer Res Treat. 2006;100 Suppl 1:S5.

70. Johnston S, Pippen J Jr, Pivot X, et al. Lapatinib combined with letrozole versus letrozole and placebo as first-line therapy for postmenopausal hormone receptor-positive metastatic breast cancer. J Clin Oncol. 2009;27(33):5538-5546.

71. Waknine Y. FDA approves lapatinib/letrozole combination for advanced breast cancer. Medscape Medical News. 2010. Available from: http://www. medscape.com/viewarticle/716263. Accessed 2010 Sep 15.

72. Storniolo AM, Pegram MD, Overmoyer B, et al. Phase I dose escalation and pharmacokinetic study of lapatinib in combination with trastuzumab in patients with advanced ErbB2-positive breast cancer. J Clin Oncol. 2008;26(20):3317-3323.

73. O'Shaughnessy J, Blackwell KL, Burstein HJ, et al. A randomized study of lapatinib alone or in combination with trastuzumab in heavily pretreated HER2+ metastatic breast cancer progressing on trastuzumab therapy. J Clin Oncol. 2008;26 No 15S:1015.

74. Blackwell K, Burstein H, Ledge G, et al. Updated survival analysis of a randomized study of lapatinib alone or in combination with trastuzumab in women with HER2-positive metastatic breast cancer progressing on trastuzumab therapy. Cancer Res. 2009;69 Suppl 24: Abstr 61.

75. Low JA, Berman AW, Steinberg SM, Danforth DN, Lippman ME, Swain SM. Long-term follow-up for locally advanced and inflammatory breast cancer patients treated with multimodality therapy. J Clin Oncol. 2004;22(20):4067-4074.

76. Chang S, Parker SL, Pham T, Buzdar AU, Hursting SD. Inflammatory breast carcinoma incidence and survival: The surveillance, epidemiology, and end results program of the National Cancer Institute, 1975-1992. Cancer. 1998;82(12):2366-2372.

77. Blackwell KL, Pegram MD, Tan-Chiu E, et al. Single-agent lapatinib for HER2-overexpressing advanced or metastatic breast cancer that progressed on first- or second-line trastuzumab-containing regimens. Ann Oncol. 2009;20(6):1026-1031.

78. Johnston S, Trudeau M, Kaufman B, et al. Phase II study of predictive biomarker profiles for response targeting human epidermal growth factor receptor 2 (HER-2) in advanced inflammatory breast cancer with lapatinib monotherapy. J Clin Oncol. 2008;26(7):1066-1072.

79. Spector NL, Blackwell K, Hurley J. EGF103009, a phase II trial of lapatinib monotherapy in patients with relapsed/refractory inflammatory breast cancer (IBC): Clinical activity and biologic predictors of response. Proc Am Soc Clin Oncol. 2006;24:3S. Abstr 502. 
80. Kaufman B, Trudeau M, Awada A, et al. Lapatinib monotherapy in patients with HER2-overexpressing relapsed or refractory inflammatory breast cancer: Final results and survival of the expanded HER2+ cohort in EGF103009, a phase II study. Lancet Oncol. 2009;10(6):581-588.

81. Cristofanilli M, Krishnamurthy S, Guerra L, et al. A phase II combination study of lapatinib and paclitaxel as a neoadjuvant therapy in patients with newly diagnosed inflammatory breast cancer (IBC). Breast Cancer Res Treat. 2006;100 Suppl 1:S5.

82. DiStefano A, Yong YY, Hortobagyi GN, Blumenschein GR. The natural history of breast cancer patients with brain metastases. Cancer. 1979;44(5):1913-1918.

83. Stewart JF, King RJ, Sexton SA, Millis RR, Rubens RD, Hayward JL. Oestrogen receptors, sites of metastatic disease and survival in recurrent breast cancer. Eur J Cancer. 1981;17(4):449-453.

84. Bria E, Cuppone F, Fornier M, et al. Cardiotoxicity and incidence of brain metastases after adjuvant trastuzumab for early breast cancer: The dark side of the moon? A meta-analysis of the randomized trials. Breast Cancer Res Treat. 2008;109(2):231-239.

85. Lin NU, Bellon JR, Winer EP. CNS metastases in breast cancer. J Clin Oncol. 2004;22(17):3608-3617.

86. Lin NU, Carey LA, Liu MC, et al. Phase II trial of lapatinib for brain metastases in patients with human epidermal growth factor receptor 2-positive breast cancer. J Clin Oncol. 2008;26(12):1993-1999.
87. Tevaarwerk AJ, Kolesar JM. Lapatinib: A small-molecule inhibitor of epidermal growth factor receptor and human epidermal growth factor receptor-2 tyrosine kinases used in the treatment of breast cancer. Clin Ther. 2009;31 Pt 2:2332-2348.

88. Tan-Chiu E, Yothers G, Romond E, et al. Assessment of cardiac dysfunction in a randomized trial comparing doxorubicin and cyclophosphamide followed by paclitaxel, with or without trastuzumab as adjuvant therapy in node-positive, human epidermal growth factor receptor 2-overexpressing breast cancer: NSABP B-31. J Clin Oncol. 2005;23(31):7811-7819.

89. Perez EA, Suman VJ, Davidson NE, et al. Exploratory analysis from NCCTG N9831: Do clinical and laboratory characteristics predict cardiac toxicity of trastuzumab when administered as a component of adjuvant therapy? Breast Cancer Res Treat. 2005;94 Suppl 1:S96.

90. Perez EA, Koehler M, Byrne J, Preston AJ, Rappold E, Ewer MS. Cardiac safety of lapatinib: Pooled analysis of 3689 patients enrolled in clinical trials. Mayo Clin Proc. 2008;83(6):679-686.

91. Red Book 2009: Pharmacy's Fundamental Reference. 113th ed. 2009.
Breast Cancer: Targets and Therapy

\section{Publish your work in this journal}

Breast Cancer: Targets and Therapy is an international, peerreviewed open access journal focusing on breast cancer research, identification of therapeutic targets and the optimal use of preventative and integrated treatment interventions to achieve improved outcomes, enhanced survival and quality of life for the cancer patient.

\section{Dovepress}

View the full aims and scopes of this journal here. The manuscript management system is completely online and includes a very quick and fair peer-review system, which is all easy to use. Visit http:// www.dovepress.com/testimonials.php to read real quotes from published authors.

Submit your manuscript here: http://www.dovepress.com/breast-cancer---targets-and-therapy-journal 